\title{
Laying down the law
}

\section{Every living thing obeys the rules of scaling discovered by Max Kleiber.}

\section{B}

Vaclav Smil $y$ the early 1930s, physics was a mature science abounding in universally applicable laws. In comparison, organismic biology was overwhelmingly descriptive and lacked quantitative expressions that could apply to a broad range of animals or plants. In 1932 Max Kleiber changed all that - he published a paper on "Body size and metabolism" in Hilgardia which included a graph plotting the log of the body weight of mammals against the log of their basal metabolic rate (BMR).

Kleiber (1893-1976) was a Swiss agricultural chemist trained at the Federal Institute of Technology in Zürich. He joined the Animal Husbandry Department at the University of California, Davis, in 1929 to study the energy metabolism of animals. Although his initial data set was rather limited, it contained mammals ranging from rats to steers - a range of body weights spanning three orders of magnitude. As BMR measures energy expenditure at rest, in a post-absorptive state (digestion increases metabolism) and in a thermoneutral environment, it conveys fundamental information about an animal's nutritional needs and allows fascinating intra- and interspecific comparisons.

Kleiber concluded that the BMR of animals depends on their body weight, $w$, as a function of $3.52 w^{0.74}$. Kleiber's exponent differed from the traditional value of 0.67 , which assumed that BMRs were a function of body surface area. Scores of species were added to the original data, extending the plot from a rat-to-steer to a mouse-to-elephant line - but the exponents of recalculated equations always came out close to 0.75 .

In his 1961 book The Fire of Life, Kleiber opted for the $3 / 4$ rule, recommending that BMR be expressed as $70 w^{0.75}$ kilocalories per day, or $3.4 w^{0.75}$ watts. As the book was widely used in university courses, often cited, and translated into German, Polish, Spanish and Japanese, the log-log mouse-to-elephant line became one of the most important and best known generalizations in bioenergetics.

Hundreds of BMRs are now available for both cold- and warm-blooded (ectothermic

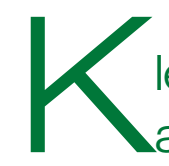

leiber's law holds across 18 orders of magnitude from microbes to whales.

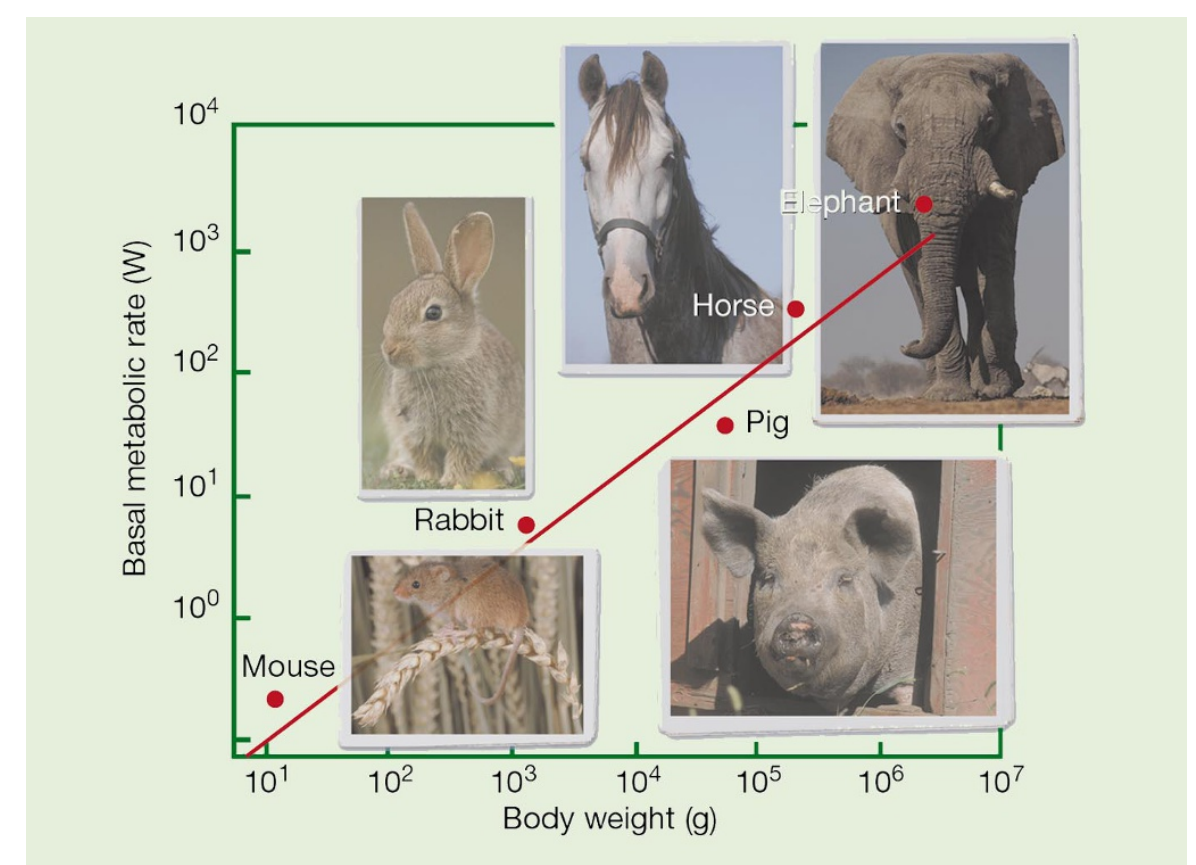

Great and small: across a vast range of animal sizes, metabolic rate is proportional to weight ${ }^{0.75}$.

and endothermic) species, and they confirm Kleiber's 3/4 law across 18 orders of magnitude, from unicellular organisms to whales. Slopes for various invertebrate groups vary from less than 0.67 to more than 1.0 , but because most of them are close enough to the 0.75 line, Knut Schmidt-Nielsen has concluded that the $3 / 4$ slope is representative for all ectotherms. While the slopes of BMRs plotted separately for various groups of organisms generally conform to the law, their positions differ substantially from values predicted by the original equation.

Predictably, the BMRs of ectotherms are only a small fraction $(2.5-5 \%)$ of those of equally massive endotherms. And among endotherms, marsupials are placed about $30 \%$ lower than eutherian mammals, whereas passerine (perching) birds are placed at least $30 \%$ above non-passerine flyers. Outlying species illustrate various modes of environmental specialization. To regulate their body temperature in cold water, seals and whales have BMRs about twice as high as other animals of their size, whereas the low BMRs of desert mammals reflect their adaptation to food shortages and to recurrent or chronic scarcity of water. Not surprisingly, sloths have relatively low BMRs, but the value for pigs takes an even greater downward departure from the prediction - and hence they make efficient meat producers.

Why the 3/4 slope? In 1973, Thomas McMahon offered an explanation based on the mechanical requirements of animal bodies. Their limb length $(L)$ is proportional to the $2 / 3$ power of muscle diameter $(d)$ and as size increases $L^{3}$ is proportional to $d^{2}$. Weight, $w$, of any limb is proportional to $L d^{2}$ and hence, by substitution, $w$ is proportional $d^{8 / 3}$. Muscle cross-section appears to be the only variable determining the maximum power of a limb - also its BMR. BMR is then proportional to $d^{2}$ or to $\left(w^{3 / 8}\right)^{2}=w^{3 / 4}$. If it is applicable to any particular muscle, the scaling should rule the whole organism and BMR should be a function of $w^{0.75}$.

An even more fundamental explanation, offered by Geoffrey West, James Brown and Brian Enquist (Science 276, 122-126; 1997), rests on the geometry and physics of the tubes that distribute resources and remove wastes in bodies. These fractal networks dictate the structural and functional properties of cardiovascular and respiratory systems in mammals and the transport of nutrients through xylem tissues in plants. Their properties require that the metabolism of organisms scales to the $3 / 4$ power of their mass. Kleiber's law is thus extended to all life forms. In contrast, Jan Kozlowski and January Weiner have concluded that the scaling patterns across species are mere by-products of the evolutionary selection that shapes body size within species. The hunt for an explanation of the 3/4 law continues.

Vaclav Smil is in the Department of Geography, University of Manitoba, Winnipeg, Canada. 